\title{
Experimental realisability of lens cloaks
}

Stephen Oxburgh, Jakub Bělín, Euan N. Cowie, Johannes Courtial

Stephen Oxburgh, Jakub Bělín, Euan N. Cowie, Johannes Courtial, "Experimental realisability of lens cloaks," Proc. SPIE 10376, Novel Optical Systems Design and Optimization XX, 103760D (24 August 2017); doi: $10.1117 / 12.2272990$

SPIE Event: SPIE Optical Engineering + Applications, 2017, San Diego, California, United States 


\title{
Experimental realisability of lens cloaks
}

\author{
Stephen Oxburgh, Jakub Bělín, Euan N. Cowie, and Johannes Courtial \\ School of Physics \& Astronomy, University of Glasgow, Glasgow G12 8QQ, Scotland
}

\begin{abstract}
We recently showed how to construct omni-directional ray-optical transformation-optics devices out of ideal thin lenses. These devices can be seen as theoretical generalisations of the paraxial, four-lens, "Rochester cloak". Here we investigate the practical realisability of such devices. We use ray-tracing simulations to compare combinations of skew lenses of different types, including ideal lenses and phase holograms of lenses.
\end{abstract}

Keywords: transformation optics; imaging; optical design; lens systems

\section{INTRODUCTION}

Our work is concerned with applications of thin light-ray-direction-changing sheets. One class of such sheets are telescope windows, which are arrays of (usually tiny) telescopes that rely on the fact that the telescopes - like the pixels of a computer screen - cannot be resolved under certain conditions, which give telescope windows the appearance of interfaces that perform generalised refraction. ${ }^{1,2}$ Telescope windows have a number of shortcomings, ${ }^{3,4}$ but they can perform very general pixellated generalised refraction, and if the shortcomings can be overcome or at least ameliorated, telescope windows also offer very general imaging. ${ }^{5,6}$ This imaging is, in fact, so general that structures of (improved) telescope windows can form transformation-optics devices. ${ }^{7,8}$

Further author information: (Send correspondence to J.C.)

J.C.: E-mail: johannes.courtial@glasgow.ac.uk, Telephone: +44 (0)141 3306429

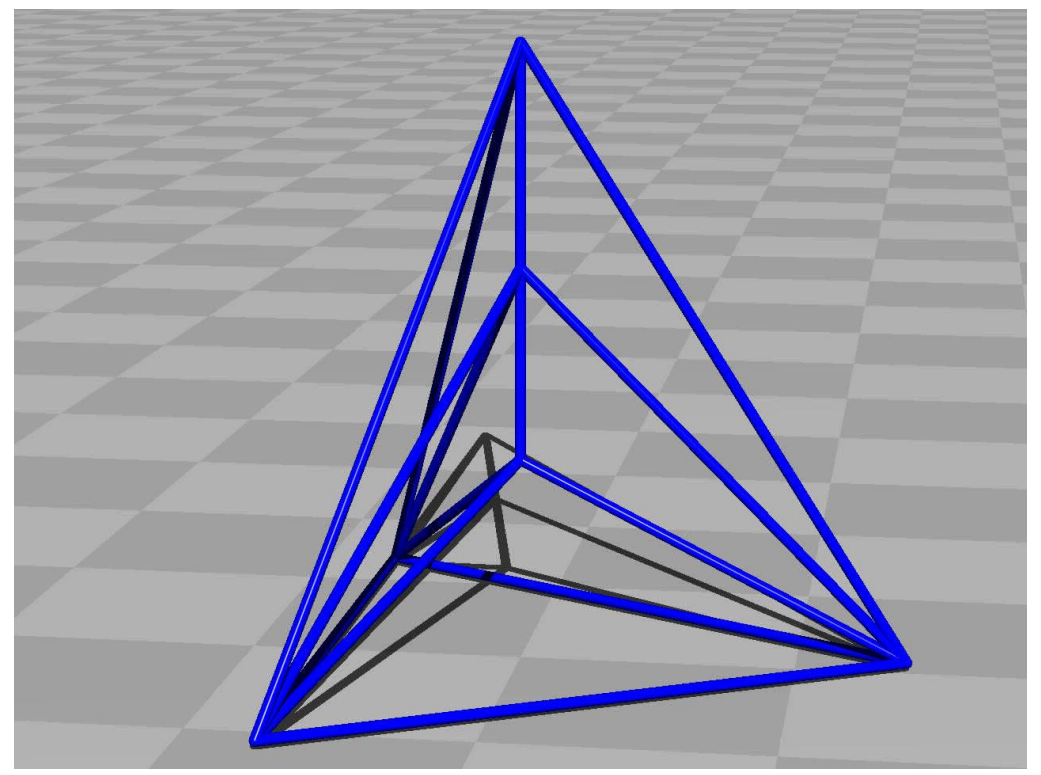

Figure 1. Ideal-lens-cloak structure. Each blue cylinder indicates an edge of a triangular ideal thin lens, and wherever three of the cylinders form a triangle, there is a suitable ideal thin lens in the ideal-lens cloak (the visual appearance of which is shown in Fig. 2). The image shown in this figure was calculated using the raytracer Dr TIM, ${ }^{9}$ and so were all other images shown in this paper. 


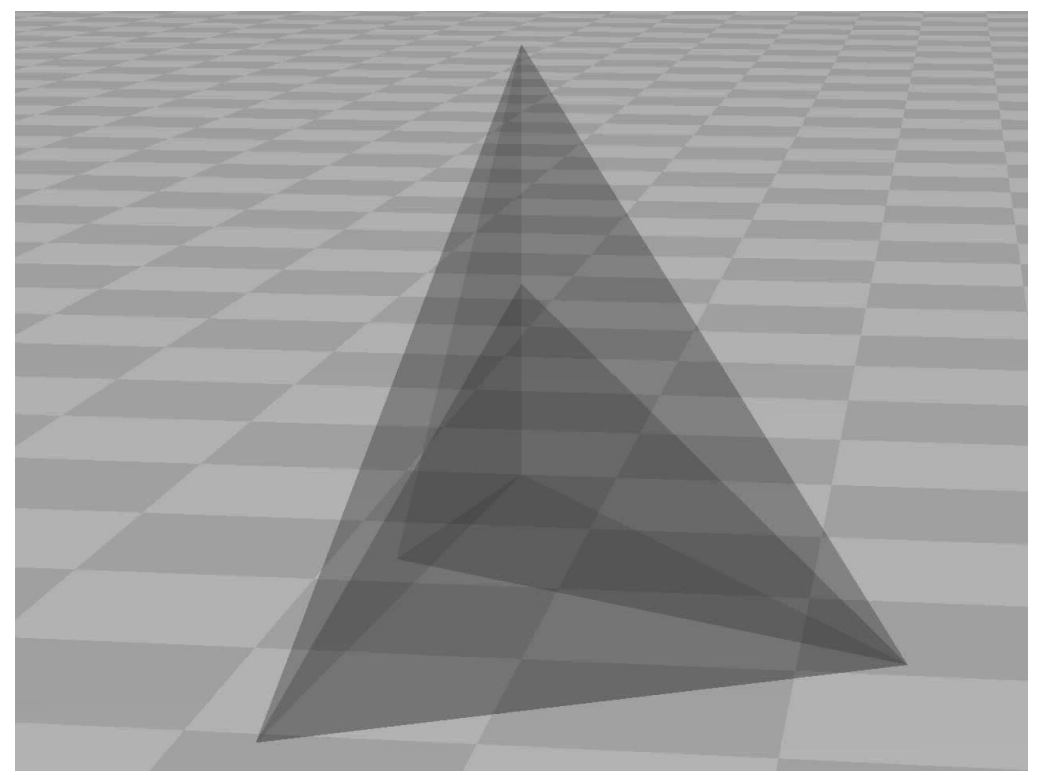

Figure 2. Visual appearance of an ideal-lens cloak. The structure of the cloak is shown in Fig. 1, and careful comparison reveals that the inner vertices appear subtly shifted (making the bottom simplex appear smaller (very slightly, in this example) than it actually is, which is the hallmark of a cloak). It can also be seen that the parts of the floor that are seen through the device are not distorted. Note that the lenses have been made absorbing to make them visible.

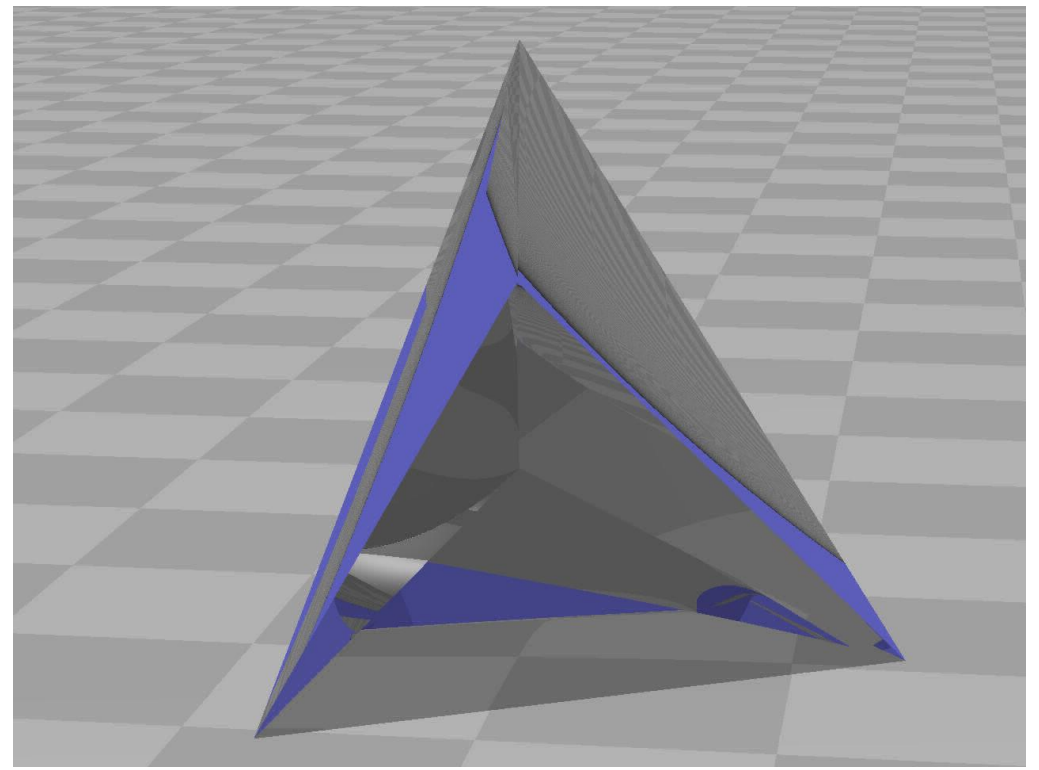

Figure 3. Visual appearance of an ideal-lens cloak. Same as Fig. 2, but with the ideal thin lenses replaced with phase holograms of lenses (each designed to do perfect axial $2 f-2 f$ imaging). Clearly, the appearance of this "lens-hologram cloak" is very different from that of the ideal-lens cloak.

Closely related to such devices are transformation-optics devices comprising entirely ideal thin lenses: in one of the telescope-window structures mentioned above, ${ }^{8}$ a few of the (improved) telescope windows are actually ideal thin lenses, and so it is natural to ask if transformation-optics devices can be constructed entirely from ideal thin lenses. It transpires that this is indeed possible. Fig. 1 shows the structure of such a device and Fig. 2 simulates its visual appearance, showing for example that the device does not distort the view through it. 


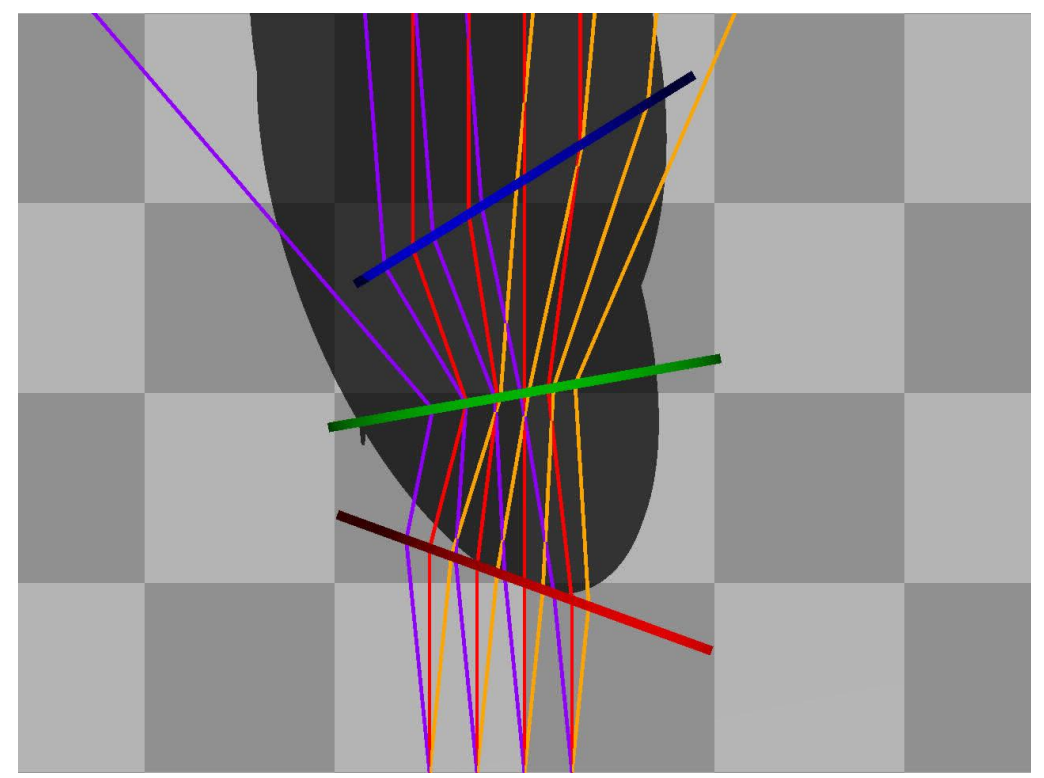

Figure 4. Top view of a combination of three skew ideal thin lenses. The view through this combination of ideal thin lenses is shown in Fig. 5. The figure also shows a number of ray trajectories through the lenses.

After successfully designing transformation-optics devices from ideal thin lenses, the obvious next step is to try to adapt the design such that it works with real lenses. However, there is a problem: the ideal-lens-cloak structure consists of combinations of skew lenses, which means that these lenses need to work not just paraxially, but also non-paraxially. Also, while real lenses can be well represented by ideal thin lenses, this is the case only when focal lengths are large and in the paraxial regime. Fig. 3 shows the ideal-lens cloak from Fig. 2 with the ideal thin lenses replaced with (still idealised) lens holograms. (We consider lens holograms instead of solid-glass lenses as these are easier to simulate in our raytracing software, Dr TIM. ${ }^{9}$ ) Clearly, the appearance of the structure has changed completely.

Here we investigate a potential way towards realising lens cloaks, but at a price: the cloak works only for a single viewer position.

\section{THE USE OF ASYMMETRIC LENS HOLOGRAMS AND FRESNEL LENSES IN SKEW-LENS COMBINATIONS}

We study combinations of skew lenses not on the full lens cloak, but on a simpler system consisting of three skew lenses. Fig. 4 shows the top view of our lens arrangement, together with a few ray trajectories that have been traced through it if each lens is an ideal thin lens. Fig. 5 shows a raytracing simulation of the view through this combination of three skew ideal thin lenses.

If the ideal thin lenses are replaced with standard lens holograms (designed such that they perform perfect on-axis $2 f-2 f$ imaging), the view through the lenses is distorted beyond recognition. This confirms that the ideal thin lenses in the ideal-lens cloak could not simply be replaced by real lenses, not without losing most, or even all, of its functionality anyway.

In the absence of ideal thin lenses, one way of realising lens cloaks is to sacrifice a few of the properties of ideal-lens cloaks. One of the properties of ideal-lens cloaks is that they work for any viewer position, and here we discuss ways to design lens cloaks that work for only one viewer position (but on the plus side, for that viewer position they work), which it is hoped will be sufficient for certain applications. This idea can be realised as follows. Phase holograms can easily be designed to image stigmatically between arbitrary conjugate positions. This is done simply by setting the phase change $\Delta \phi$ at each point on the hologram such that sum of the the phase changes experienced by a light ray to travel from the first position to the point on the hologram, and that 


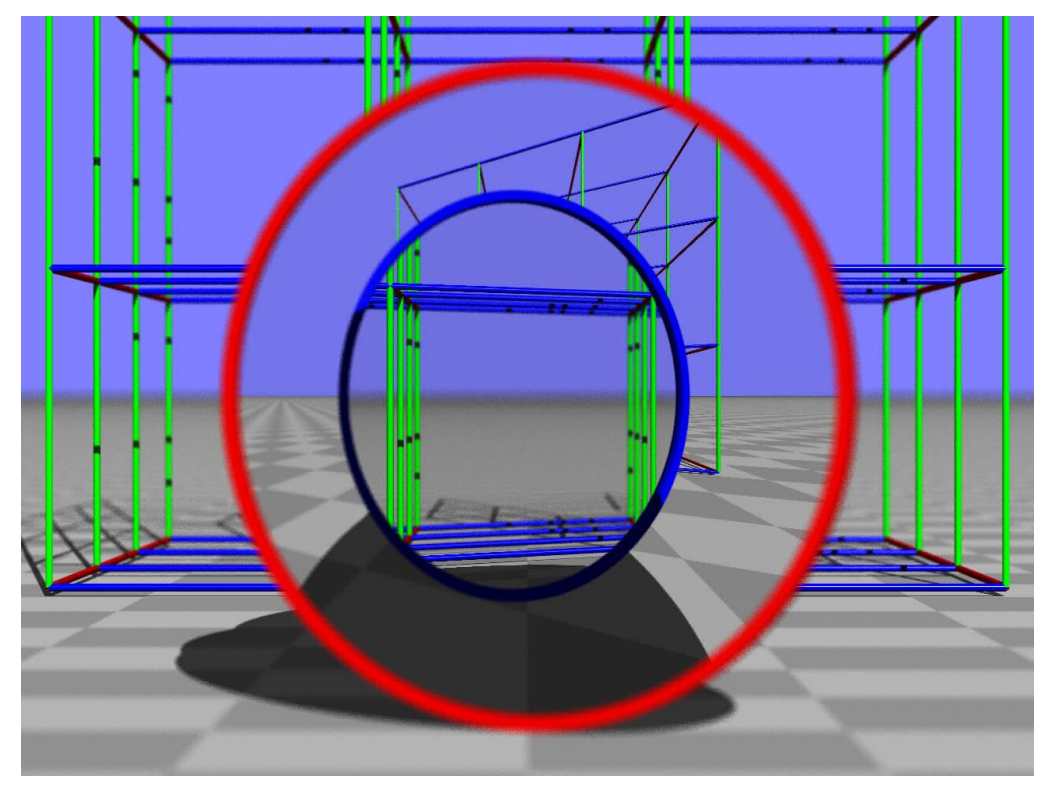

Figure 5. View through the combination of three skew ideal thin lenses shown in Fig. 4. The lens closest to the camera has a red rim, the next closest lens has a green rim, the farthest lens has a blue rim.

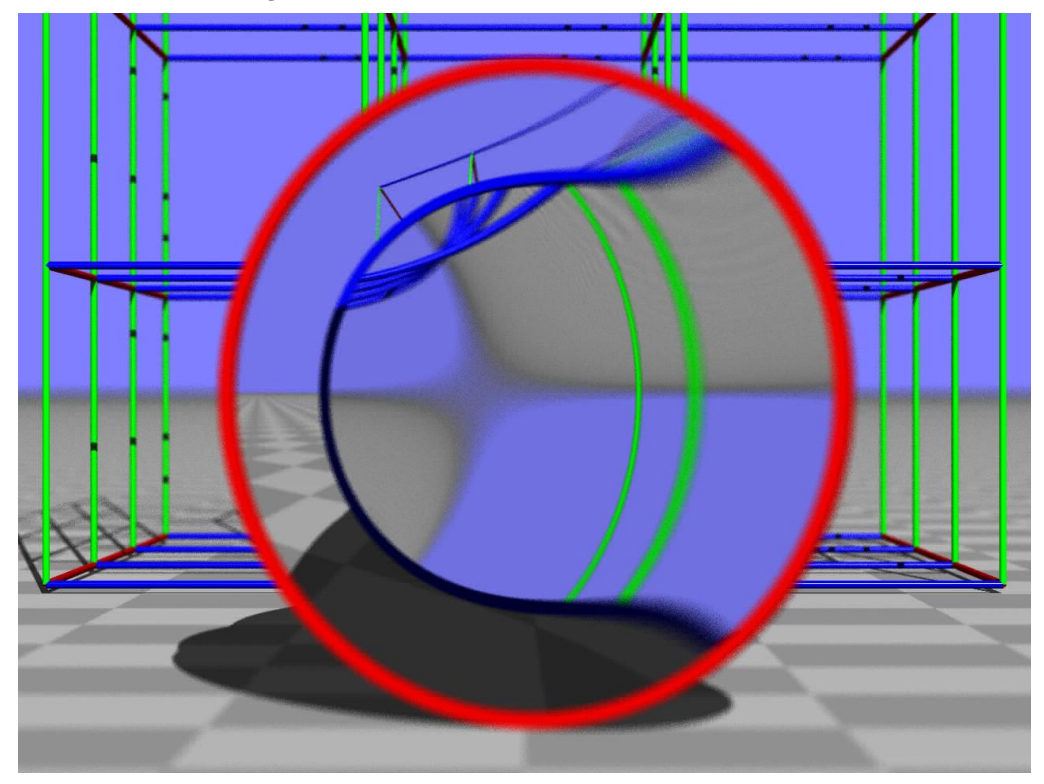

Figure 6. View through the same setup as in Fig. 4, but with the ideal thin lenses replaced with symmetric lens holograms. The holograms were optimised for imaging of an on-axis position a distance $2 f$ in front of the lens to an on-axis position the same distance behind the lens. The view through the lenses is unrecognisably distorted.

experienced by a light ray to travel from that point on the hologram to the second position, and $\Delta \phi$, which is the phase change experienced by the light ray to pass through the hologram at the position, is the same irrespective of the point on the hologram the light ray passed through. The lens holograms simulated in Fig. 6 were designed such that they image between axial positions a distance $2 f$ on either side of the hologram. We propose to alter the hologram design such that it images between suitable images of the viewer position.

Specifically, the lens closest to the viewer is designed to image the viewer position stigmatically to the position of the position of the viewer position's image the analogous ideal thin lens would produce; the lens next closest 


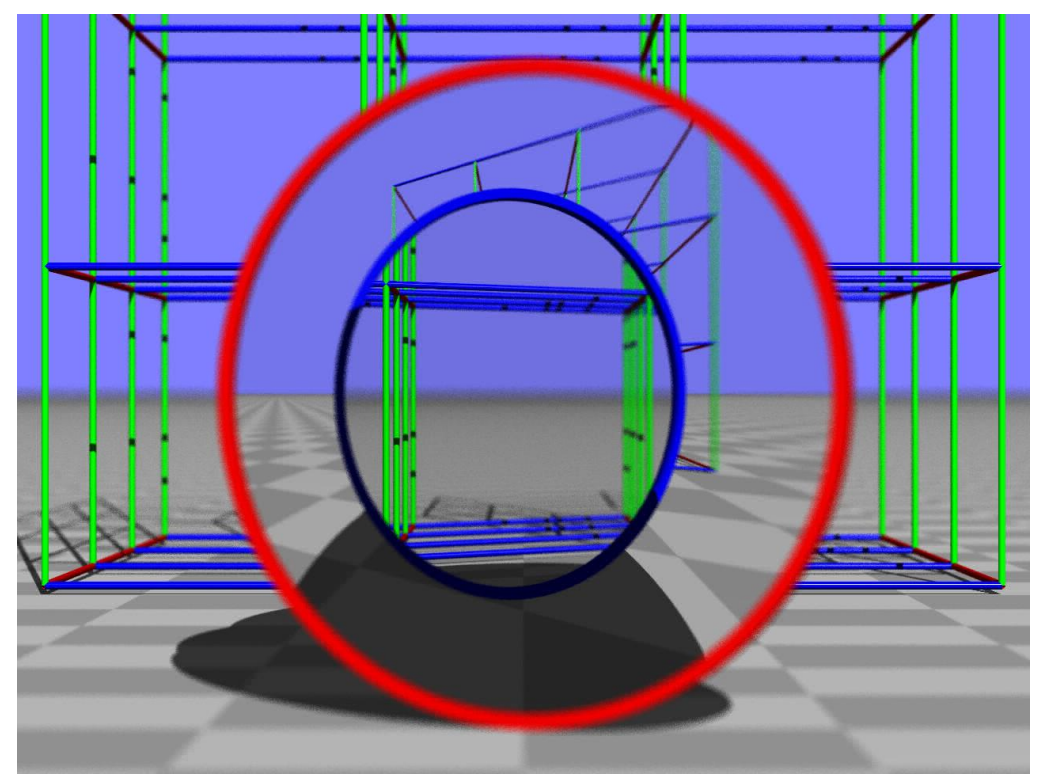

Figure 7. View through the same setup as in Fig. 6, but with the phase holograms designed such that they image stigmatically between suitable images of the viewer position.

to the viewer images this position to its image its analogous ideal thin lens would produce; and so forth. In this way, all rays that are seen by the viewer, i.e. which actually reach the viewer position, are redirected by the lens holograms exactaly as they would by an ideal thin lens.

Fig. 7 demonstrates that this idea works, at least in the computer: the view through the holograms is almost exactly that through the corresponding ideal thin lenses, shown in Fig. 5. The small differences (such as blurring of parts of the lattice) are due to the finite aperture of the simulated camera.

We note that this proposed new application for phase holograms comes at a time when phase holograms, realised in the form of metasurfaces, are undergoing rapid advances. ${ }^{10-15}$ Excitingly, such a realisation offers new possibilities, including partial dispersion compensation. ${ }^{16}$ Metasurfaces have specifically been used to realised improved lens holograms, ${ }^{17-19}$ with further improvements expected.

Despite the promises of metasurface-based phase holograms, a realisation that uses simple refraction would be desirable for a number of reasons, including ease of manufacturability using existing techniques. For this reason, we also consider here a realisation in terms of glass surfaces. The two surfaces of a lens can be optimised to image, stigmatically, between two arbitrary positions, which is precisely what we require, but this can result in a thick lens, which is problematic for our application.

Therefore, we investigate here instead a realisation in terms of Fresnel lenses that image stigmatically between two arbitrary positions, one in the space of light rays that travel on the front of the lens, the other in the space of light rays travelling behind the lens. We design the front surface of our Fresnel lenses such that it redirects light rays from the conjugate position in the space of light rays that travel on the front of the lens to become parallel to the straight line between the two conjugate positions, and the back surface such that it makes those light rays converge on the other conjugate position. Fig. 8 shows a side view of one such Fresnel-lens surface, with a few light rays traced through it; Fig. 9 shows a front view.

Fig. 10 shows the view through the three-lens combination shown in Fig. 4, but with the ideal thin lenses replaced with Fresnel lenses optimised to image between images of the viewer position. In other words, the Fresnel lenses in Fig. 10 are the Fresnel-lens equivalents of the phase holograms shown in Fig. 7. It can be seen that the concept works: the view through the lens combination is recognisably the same as that through the corresponding ideal thin lenses (Fig. 5), but with imperfections that include absorption (in particular just inside the left edge of the front lens), a slight offset of the images at the boundary between different surface sections 


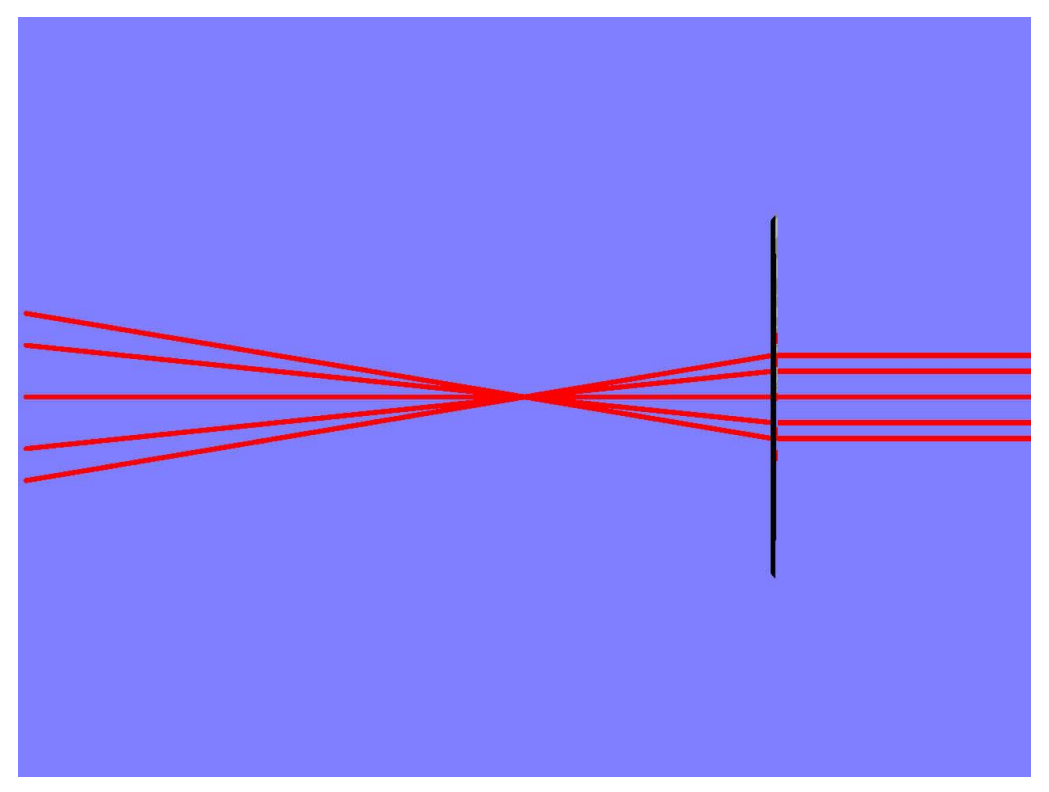

Figure 8. Side view of light rays (red lines) passing through a Fresnel-lens surface that redirects light rays from a point into a common direction. The image is a raytracing simulation through the detailed structure of the surface, whose appearance from the front is shown in Fig. 9.

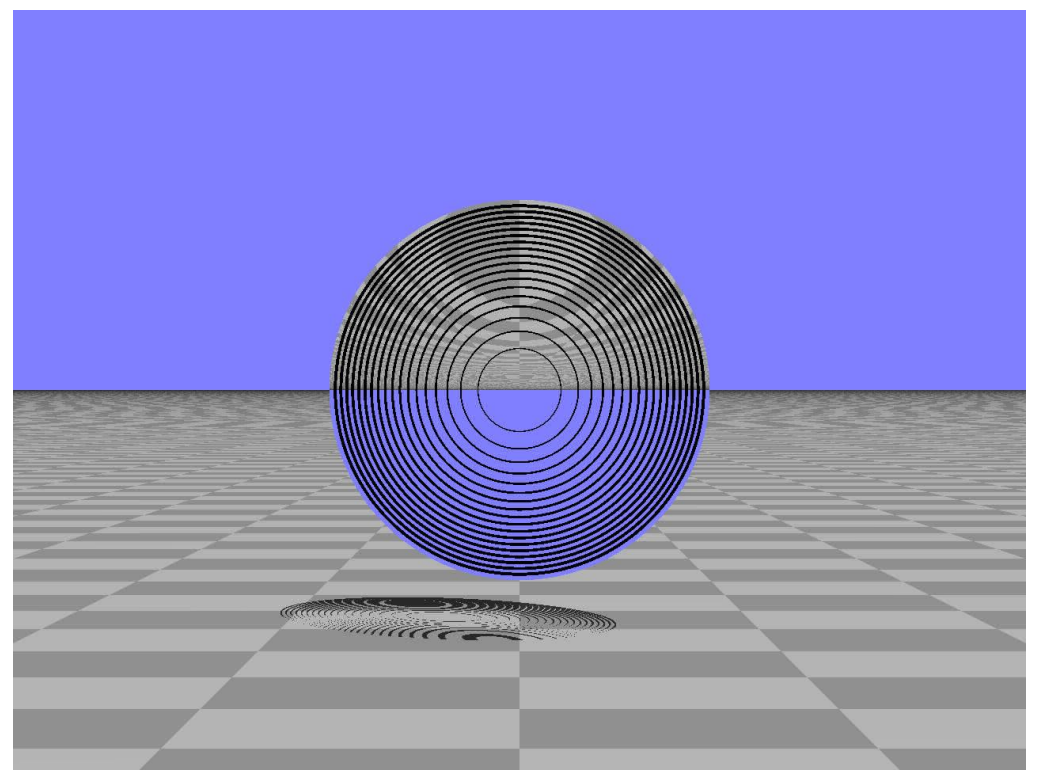

Figure 9. View through the Fresnel-lens surface shown in Fig. 8. The black circles are the steps in the surface, which have been blackened to avoid light that is wrongly redirected.

(due to the finite thickness of the Fresnel lenses), and the slight difference in blurring that was already observed in Fig. 7.

\section{CONCLUSIONS}

We have discussed and illustrated issues in the realisation of ideal-lens cloaks in terms of real lenses. We have also suggested a compromise that enables such devices to work but only for a single viewer position. 


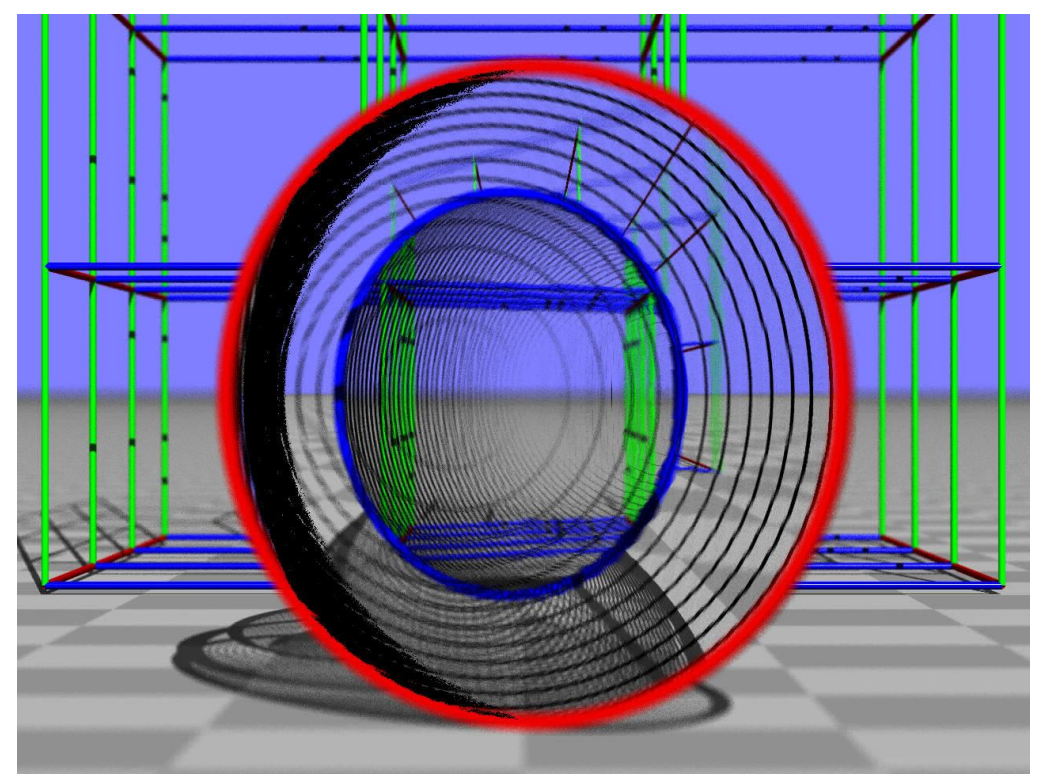

Figure 10. View through the three-lens combination shown in Fig. 4, but with the ideal thin lenses replaced with Fresnel lenses designed such that they image stigmatically between suitable images of the viewer position. This figure is the Fresnel-lens equivalent of Fig. 7.

\section{ACKNOWLEDGMENTS}

We would like to thank Miguel Alonso (Rochester) for very useful discussions. This work was supported by the Engineering and Physical Sciences Research Council [grant numbers EP/K503058/1 and EP/M010724/1].

\section{REFERENCES}

[1] J. Courtial, "Ray-optical refraction with confocal lenslet arrays," New J. Phys. 10, p. 083033, 2008.

[2] A. C. Hamilton and J. Courtial, "Generalized refraction using lenslet arrays," J. Opt. A: Pure Appl. Opt. 11, p. 065502, 2009.

[3] J. Courtial, "Standard and non-standard metarefraction with confocal lenslet arrays," Opt. Commun. 282, pp. 2634-2641, 2009.

[4] T. Maceina, G. Juzeliūnas, and J. Courtial, "Quantifying metarefraction with confocal lenslet arrays," Opt. Commun. 284, pp. 5008-5019, 2011.

[5] J. Courtial, "Geometric limits to geometric optical imaging with infinite, planar, non-absorbing sheets," Opt. Commun. 282, pp. 2480-2483, 2009.

[6] S. Oxburgh and J. Courtial, "Perfect imaging with planar interfaces," J. Opt. Soc. Am. A 30, pp. 2334-2338, 2013.

[7] S. Oxburgh, C. D. White, G. Antoniou, E. Orife, T. Sharpe, and J. Courtial, "Large-scale, white-light, transformation optics using integral imaging," J. Opt. 18, p. 044009, 2016.

[8] T. Tyc, S. Oxburgh, E. N. Cowie, G. J. Chaplain, G. Macauley, C. D. White, and J. Courtial, "Omnidirectional transformation-optics cloak made from lenses and glenses," J. Opt. Soc. Am. A 33, pp. 10321040, 2016.

[9] S. Oxburgh, T. Tyc, and J. Courtial, "Dr TIM: Ray-tracer TIM, with additional specialist capabilities," Comp. Phys. Commun. 185, pp. 1027-1037, 2014.

[10] N. Yu, P. Genevet, M. A. Kats, F. Aieta, J.-P. Tetienne, F. Capasso, and Z. Gaburro, "Light propagation with phase discontinuities: Generalized laws of reflection and refraction," Science 334, pp. 333-337, 2011.

[11] X. Ni, A. V. Kildishev, and V. M. Shalaev, "Metasurface holograms for visible light," Nature Communications 4, p. 2807, 2013. 
[12] N. Yu and F. Capasso, "Flat optics with designer metasurfaces," Nat. Mater. 13, p. 139, 2014.

[13] G. Zheng, H. Mühlenbernd, M. Kenney, G. Li, T. Zentgraf, and S. Zhang, "Metasurface holograms reaching 80\% efficiency," Nat. Nanotech. 10, p. 308, 2015.

[14] L. Wang, S. Kruk, H. Tang, T. Li, I. Kravchenko, D. N. Neshev, and Y. S. Kivshar, "Grayscale transparent metasurface holograms," Optica 3, pp. 1504-1505, 2016.

[15] P. Genevet, F. Capasso, F. Aieta, M. Khorasaninejad, and R. Devlin, "Recent advances in planar optics: from plasmonic to dielectric metasurfaces," Optica 4, pp. 139-152, 2017.

[16] F. Aieta, M. A. Kats, P. Genevet, and F. Capasso, "Multiwavelength achromatic metasurfaces by dispersive phase compensation," Science 347, pp. 1342-1345, 2015.

[17] M. Khorasaninejad, F. Aieta, P. Kanhaiya, M. A. Kats, P. Genevet, D. Rousso, and F. Capasso, "Achromatic metasurface lens at telecommunication wavelengths," Nano Lett. 15, pp. 5358-5362, 2015.

[18] E. Arbabi, A. Arbabi, S. M. Kamali, Y. Horie, and A. Faraon, "Multiwavelength polarization-insensitive lenses based on dielectric metasurfaces with metamolecules," Optica 3, p. 628, 2016.

[19] M. Khorasaninejad, W. T. Chen, R. C. Devlin, J. Oh, A. Y. Zhu, and F. Capasso, "Metalenses at visible wavelengths: Diffraction-limited focusing and subwavelength resolution imaging," Science 352, pp. 11901194, 2016. 